\title{
Assessment of Biological Reaction to Whole Body Vibration Training by Evaluating Changes in Salivary Components and Cutaneous Blood Flow
}

\author{
Seiko Yamaguchi ${ }^{*}$, Yousuke Morita ${ }^{1}$, Takehiko Yukishita1, Keiko K. Lee ${ }^{2}$, \\ Takuji Yamaguchi'2, Naoko Koga1, Hiroyuki Kobayashi,,2 \\ ${ }^{1}$ Department of Hospital Administration, Juntendo University Graduate School of Medicine, Tokyo, Japan \\ ${ }^{2}$ Center for Advanced Kampo Medicine and Clinical Research, Juntendo University Graduate School of \\ Medicine, Tokyo, Japan \\ Email: seiko-y@juntendo.ac.jp
}

Received 5 March 2014; revised 7 April 2014; accepted 15 April 2014

Copyright @ 2014 by authors and Scientific Research Publishing Inc.

This work is licensed under the Creative Commons Attribution International License (CC BY).

http://creativecommons.org/licenses/by/4.0/

\section{Open Access}

\begin{abstract}
Aim: Whole body vibration (WBV) is thought to improve blood flow and autonomic balance and thereby induce a relaxation effect, which suggests its use for stress management. However, the relaxation effect of WBV training has not been objectively evaluated thus far. The purpose of this study was to determine the biological response to WBV training by measuring peripheral blood flow and salivary components using non-invasive techniques. Methods: Participants included 10 healthy volunteers ( 7 men, 3 women; mean age $33.8 \pm 2.3$ ) who provided oral consent and served as their own control. Each participant performed 15 types of stretching exercises for $10.5 \mathrm{~min}$ on the Power Plate ${ }^{\circledR}$ and cutaneous blood flow and salivary components were measured before and after the exercise. One week later, all participants performed the same exercise regimen for 10.5 min on a non-vibratory plate, and blood flow measurement and salivary tests were performed in a similar manner. Cutaneous blood flow was measured in the 4th digit for 1 min using the laser speckle flowgraphy. Saliva samples were evaluated for cortisol levels and $\alpha$-amylase activity. To determine the effects of stretching exercises on the Power Plate ${ }^{\circledR}$ vs a non-vibratory plate, the differences in pre- and post-exercise peripheral blood flow, salivary cortisol levels, and salivary $\alpha$-amylase activity were statistically evaluated by the t-test. Results: Mean blood flow before and after the exercise on the Power Plate ${ }^{\circledR}$ was $122.0 \pm 54.2$ and $156.7 \pm 51.2$, respectively; on a non-vibratory plate, blood flow was $136.6 \pm 47.9$ and $146.3 \pm 38.3$, respectively. The differences in pre-exercise and post-exercise values of the two training methods were not significant $(p=0.215)$.
\end{abstract}

\footnotetext{
*Corresponding author.
} 
Mean cortisol levels before and after the exercise on the Power Plate ${ }^{\circledR}$ were $266.6 \pm 125$ and 204.9 \pm 61.6 , respectively; on a non-vibratory plate, the levels were $439.0 \pm 121.7$ and $425.8 \pm 118.8$, respectively. The differences in pre-exercise and post-exercise values of the two training methods were not significant $(p=0.384)$. Mean $\alpha$-amylase activity before and after the exercise on the Power Plate ${ }^{\circledR}$ was $3.74 \pm 2.89$ and $5.40 \pm 3.76$, respectively; on a non-vibratory plate, the activity was $3.95 \pm 2.23$ and $3.28 \pm 1.73$. The differences in pre-exercise and post-exercise values of the two training methods were not significant $(p=0.115)$. Conclusion: Our results showed that a brief WBV training increased peripheral blood flow, reduced cortisol levels, and increased $\alpha$-amylase activity. WBV appears to regulate autonomic activity, in particular, suppress sympathetic activity and improve bodily functions. Thus WBV exercise may be conductive for stress management, but further investigation is warranted to determine the optimal duration of WBV training for stress relief.

\section{Keywords}

Whole Body Vibration, Cutaneous Blood Flow, Salivary Cortisol, Salivary $\alpha$-Amylase Activity, Relaxation Effect

\section{Introduction}

Whole body vibration (WBV) training confers the benefits of weight training under minimal effects of gravity through the delivery of accelerating load from an oscillating device [1]. The reported benefits of WBV training in the elderly include enhanced balance, muscular strength [2] [3], and improved bone density in conditions such as osteoporosis through the application of a mechanical load [4] [5]. Other reported benefits of WBV training include reduction of muscular hypertonicity, strengthening of lower extremities, and improvement in gait particularly in patients with stroke [6] [7], spastic paralysis [8], multiple sclerosis [9], and Parkinson's disease [10][12].

Meanwhile, WBV training appears to have a relaxing effect as well. Physical stimulation of the muscles in WBV training has been speculated to improve blood flow and autonomic balance and thereby induce a relaxation effect, which may be helpful as a form of stress management for the elderly and patients with physical impairments. The WBV device occupies minimal space and can be easily used by patients with physical limitations. Many patients could derive significant benefits by improving both their physical and psychological health through a brief WBV session in a hospital setting if the device was easily accessible.

However, the relaxation effect of WBV training has never been thoroughly investigated. Thus, this study was conducted to objectively determine the biological response to WBV training by evaluating cutaneous blood flow and salivary cortisol and $\alpha$-amylase levels. Given that peripheral blood flow and stress markers are best measured using minimally invasive techniques, we used the noninvasive laser speckle flowgraphy and saliva testing for our evaluation.

\section{Participants and Methods}

\subsection{Participants}

This study was conducted as a within subject design wherein each participant served as his/her own control. Participants included 10 healthy volunteers ( 7 men, 3 women; mean age $33.8 \pm 2.3$ ) who provided verbal consent to participate in the study. Those with difficulty in performing the WBV exercise, with cardiovascular disorders such as hypertension, hyperlipidemia, or diabetes, on drugs that affect autonomic functions, or are pregnant or have the possibility of becoming pregnant were excluded from the study.

\subsection{Methods}

All participants performed 15 types of stretching exercises consecutively for $10.5 \mathrm{~min}$ on a WBV platform (Power Plate ${ }^{\circledR}$; PROTEA JAPAN, Tokyo). The different types of stretching exercises and the time required to perform each of them are described in Table 1 and Figure 1. 
Table 1 . Whole body vibration stretch menu.

\begin{tabular}{|c|c|c|c|c|c|}
\hline Position & Exercise & Time (second) & Frequency (Hz) & Strength $(\mathrm{H} / \mathrm{L})$ & Number of set \\
\hline 1 & Hamstring stretch & 30 & 30 & $\mathrm{~L}$ & 1 \\
\hline 2 & Quad stretch & 30 & 30 & $\mathrm{~L}$ & 1 (each leg) \\
\hline 3 & Adductor stretch & 30 & 30 & $\mathrm{~L}$ & 1 (each leg) \\
\hline 4 & Calf stretch & 30 & 30 & $\mathrm{~L}$ & 1 \\
\hline 5 & Squat & 30 & 30 & $\mathrm{~L}$ & 1 \\
\hline 6 & Wide-stance squat & 30 & 30 & $\mathrm{~L}$ & 1 \\
\hline 7 & Pelvic bridge & 30 & 30 & $\mathrm{~L}$ & 1 \\
\hline 8 & Push-up & 30 & 30 & $\mathrm{~L}$ & 1 \\
\hline 9 & Triceps & 30 & 30 & $\mathrm{~L}$ & 1 \\
\hline 10 & Crunch & 30 & 30 & $\mathrm{~L}$ & 1 \\
\hline 11 & Front plank & 30 & 30 & $\mathrm{~L}$ & 1 \\
\hline 12 & Lateral plank & 30 & 30 & $\mathrm{~L}$ & 1 (each arm) \\
\hline 13 & Hamstring massage & 60 & 35 & $\mathrm{H}$ & 1 \\
\hline 14 & Quad massage & 60 & 35 & $\mathrm{H}$ & 1 \\
\hline 15 & Back relaxer & 60 & 30 & $\mathrm{~L}$ & 1 \\
\hline
\end{tabular}

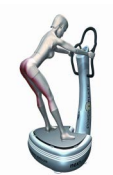

Hamstring stretch

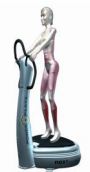

Calf stretch

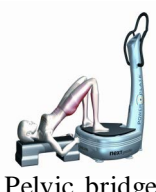

Pelvic bridge
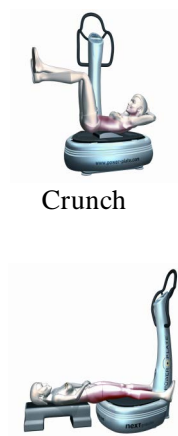

Hamstring massage

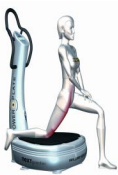

Quad stretch

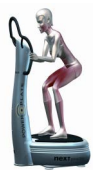

Squat
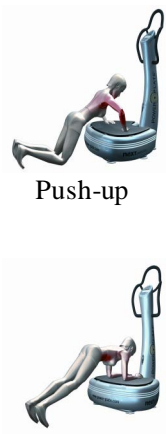

Front plank

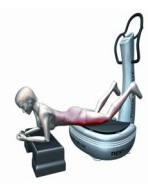

Quad massage

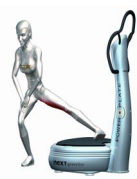

Adductor stretch

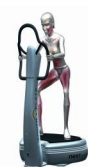

Wide-stance squat
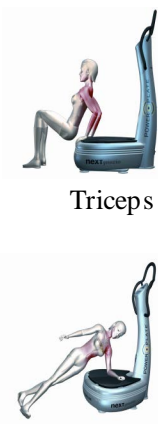

Lateral plank

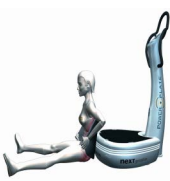

Back relaxer

\section{Figure 1. Whole body vibration stretch menu.}


Cutaneous blood flow and salivary samples were collected before and after the exercise. One week later, all the participants performed the same exercise regimen consecutively on a non-oscillating plate for $10.5 \mathrm{~min}$, and cutaneous blood flow and saliva components were also measured before and after the exercise. All the tests and measurements were taken before 11:00 to account for diurnal changes in heart rate variability and performed in a testing room maintained between $24^{\circ} \mathrm{C}$ and $26^{\circ} \mathrm{C}$.

\subsection{Cutaneous Blood Flow}

Laser speckle flowgraphy (Moor FLPI, Moor Instruments, Essex, UK) is a noninvasive device that measures blood flow in real-time without direct contact with the participant. The imager measures blood flow through delivering laser light to the biological tissues and capturing speckle patterns over time. Participants sat quietly while cutaneous blood flow in the 4th digit of the right hand was recorded for 1 min using the laser speckle flowgraphy.

\subsection{Saliva Collection}

Patients retained an odorless and tasteless swab (Salimetrics Oral Swab, Salimetrics LLC, USA) in their mouth for $5 \mathrm{~min}$ in a seated position. Swabs were removed and saliva samples were centrifuged at $1500 \mathrm{rpm}$ for $30 \mathrm{~min}$ at $4^{\circ} \mathrm{C}$. Samples were stored at $-20^{\circ} \mathrm{C}$ until analysis since it has been reported that the concentration of saliva samples can be stored long term in a freezer without inducing compositional changes [13].

\subsection{Salivary Component Analysis}

Salivary cortisol levels and $\alpha$-amylase activity were measured using the collected samples. Cortisol levels were measured using the Cortisol EIA Kit (Enzo Life Sciences, USA). $\alpha$-Amylase activity was determined using the Salivary $\alpha$-Amylase Assay Kit (Salimetrics, USA), and salivary protein levels were used as a proxy in estimating salivary production using the Pierce BCA Protein Assay (Thermo Scientific, Rockford, USA). The $\alpha$-amylase activity was corrected for flow rates by dividing the activity by the amount of salivary production.

\subsection{Statistical Analysis}

To compare the effects of stretching exercise performed on the Power Plate ${ }^{\circledR}$ vs a non-vibratory plate, pre-exercise values of cutaneous blood flow, salivary cortisol levels, and salivary $\alpha$-amylase activity were subtracted from the post-exercise values to determine the magnitude of change in these parameters. The $t$-test was performed to compare the differences in values obtained during stretching on Power Plate ${ }^{\circledR}$ vs a non-vibratory plate. Significance was set at 5\% for both analyses. The SPSS Ver. 11.5 software was used to perform data analysis.

\section{Results}

\subsection{Cutaneous Blood Flow}

Table 2 and Figure 2 show the mean and standard deviations of pre-exercise and post-exercise blood flow and the difference between the two values. Mean blood flow before and after stretching exercise on the Power Plate ${ }^{\circledR}$ was $122.0 \pm 54.2$ and $156.7 \pm 51.2$, respectively, with a difference of $34.7 \pm 42.9$. By contrast, blood flow before and after stretching on a non-vibratory plate was $136.6 \pm 47.9$ and $146.3 \pm 38.3$, respectively, with a difference of $9.7 \pm 31.6$. The changes in pre-exercise and post-exercise values (Power Plate ${ }^{\circledR}$ vs non-vibratory plate) were not significant $(\mathrm{p}=0.215)$.

Table 2. Effects of power plate on cutaneous blood flow and salivary components.

\begin{tabular}{|c|c|c|c|c|c|c|c|}
\hline & \multicolumn{3}{|c|}{ Power plate + stretch } & \multicolumn{3}{|c|}{ Stretch } & \multirow{3}{*}{ p-value } \\
\hline & Pre-test & Post-test & Change & Pre-test & Post-test & Change & \\
\hline & Mean \pm SD & Mean \pm SD & Mean \pm SD & Mean \pm SD & Mean \pm SD & Mean \pm SD & \\
\hline Blood flow & $122.0 \pm 54.2$ & $156.7 \pm 51.2$ & $34.65 \pm 42.9$ & $136.6 \pm 47.9$ & $146.3 \pm 38.3$ & $9.7 \pm 31.6$ & 0.215 \\
\hline Cortisol & $266.6 \pm 125.5$ & $204.9 \pm 61.6$ & $-61.5 \pm 95.0$ & $439.0 \pm 121.7$ & $425.8 \pm 118.8$ & $-13.2 \pm 102.9$ & 0.38 \\
\hline$\alpha$-Amylase & $3.74 \pm 2.89$ & $5.40 \pm 3.76$ & $1.66 \pm 4.18$ & $3.95 \pm 2.23$ & $3.28 \pm 1.73$ & $-0.67 \pm 3.59$ & 0.11 \\
\hline
\end{tabular}




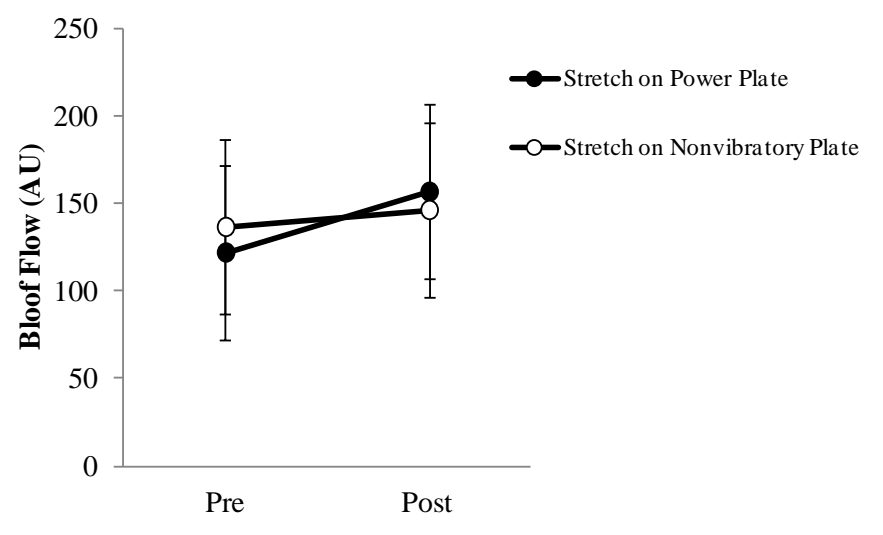

Figure 2. Cutaneous blood flow before and after stretching exercise on power plate vs non-vibratory plate.

\subsection{Salivary Components}

\subsubsection{Cortisol Levels}

Table 2 and Figure 3 show the mean and standard deviations of pre-exercise and post-exercise cortisol levels and the difference between the two values. Mean cortisol levels before and after stretching exercise on the Power Plate ${ }^{\circledR}$ were $266.6 \pm 125$ and $204.9 \pm 61.6$, respectively, with a difference of $-61.5 \pm 95.0$. By contrast, mean cortisol levels before and after stretching exercise on a non-vibratory plate were $439.0 \pm 121.7$ and $425.8 \pm$ 118.8 , respectively, with a difference of $-13.2 \pm 102.9$. The changes in pre-exercise and post-exercise values (Power Plate ${ }^{\circledR}$ vs non-vibratory plate) were not significant $(\mathrm{p}=0.384)$.

\subsection{2. $\alpha$-Amylase Activity}

Table 2 and Figure 4 show the mean and standard deviations of pre-exercise and post-exercise of $\alpha$-amylase activity and the difference between the two values. Mean $\alpha$-amylase activity before and after stretching exercise on the Power Plate ${ }^{\circledR}$ was $3.74 \pm 2.89$ and $5.40 \pm 3.76$, respectively, with a difference of $1.66 \pm 4.18$. By contrast, mean $\alpha$-amylase activity before and after stretching exercise on a non-vibratory plate was $3.95 \pm 2.23$ and $3.28 \pm$ 1.73 , respectively, with a difference of $-0.67 \pm 3.59$. The changes in pre-exercise and post-exercise values (Power Plate ${ }^{\circledR}$ vs non-vibratory plate) were not significant $(\mathrm{p}=0.115)$.

\section{Discussion}

Our results show that whole body vibration delivered for 10.5 min on a Power Plate ${ }^{\circledR}$ improved cutaneous blood flow, decreased salivary cortisol levels, and increased salivary $\alpha$-amylase activity. These findings indicate that WBV training on a Power Plate ${ }^{\circledR}$ generates a contradictory activation and inhibition of the sympathetic activity.

After exercising on the Power Plate ${ }^{\circledR}$, participants had increased cutaneous blood flow, which is suggestive of the inhibition of sympathetic activity because peripheral blood flow reflects the sole regulation of blood vessels by the sympathetic nerves. Under normal circumstances, sympathetic nervous activation results in peripheral vasoconstriction and consequent reduction in blood flow. By contrast, inhibition of sympathetic nerves results in peripheral vasodilation and resultant increased blood flow.

Further, we surmised that a reduction in salivary cortisol levels after stretching could be attributed to the inhibition of sympathetic regulation as well. Salivary cortisol reflects the amount of cortisol secreted into the bloodstream by the adrenal glands in response to adrenocorticotropic hormone release from the pituitary gland. Basco et al. has reported that plasma cortisol levels decrease after WBV training [14], which is in line with our findings from this study.

On the other hand, salivary $\alpha$-amylase activity increased among the participants after training on the Power Plate ${ }^{\circledR}$, which is indicative of sympathetic dominance. Although significant differences in pre-exercise and postexercise values in response to training on the Power Plate ${ }^{\circledR}$ vs non-vibratory plate were not found, salivary $\alpha$-amylase activity decreased after stretching on the non-vibratory plate. $\alpha$-Amylase is secreted under the influence of both the sympathetic nervous-adrenal medullary (SAM) system (direct nerve activation) and norepinephrine, i.e., under stressful conditions. 


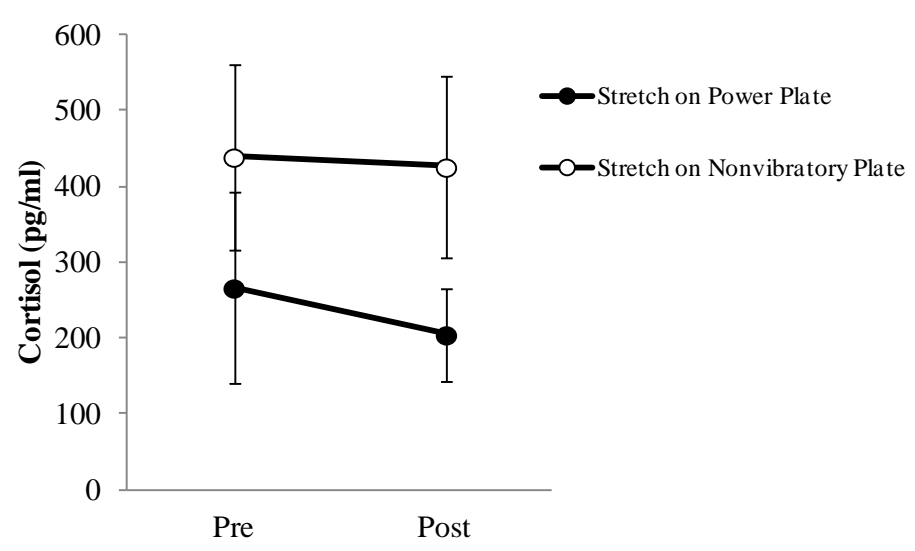

Figure 3. Salivary cortisol levels before and after stretching exercise on power plate vs non-vibratory plate.

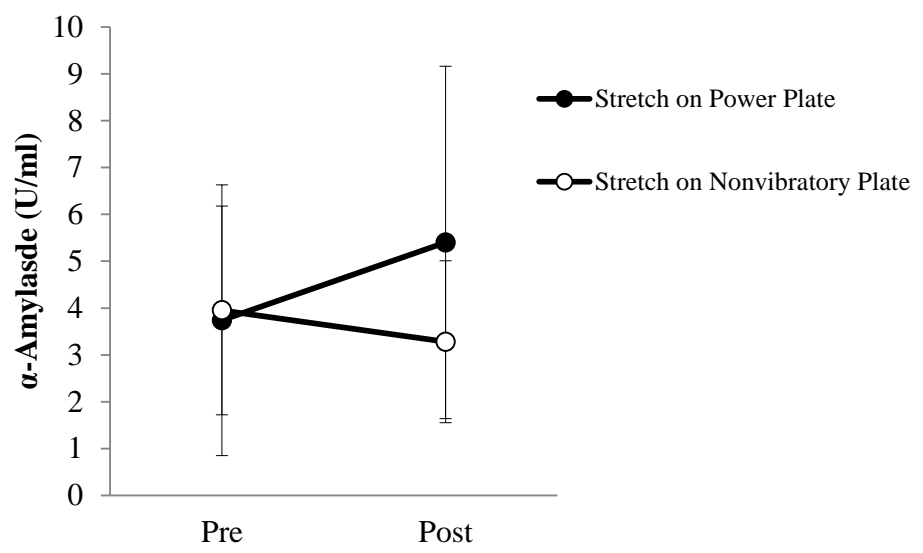

Figure 4. Salivary $\alpha$-amylase activity before and after stretching exercise on power plate vs non-vibratory plate.

Overall we found an intriguing simultaneous activation and inhibition of sympathetic activity. The contradictory sympathetic activity after Power Plate ${ }^{\circledR}$ exercise may be explained by the difference in response times of the measured parameters. The biological stress defense mechanisms include the hypothalamic-pituitary-adrenocortical (HPA) system and the SAM system. The HPA axis is the endogenous, primary mechanism that is activated in stress response to cope with external demands [15]. By contrast, the SAM system is an acute autonomic response first coined as the "fight or flight" response by Cannon (1935) [16].

Peripheral blood flow is under the sole influence of sympathetic activity and is considered to reflect stress response mediated by the SAM system. In other words, peripheral blood flow is hindered when stress is high due to sympathetic dominance, but the precise response time using the laser speckle flowgraphy has not been reported to date.

Cortisol is a hormone that is released into the blood in response to HPA activation, thus salivary and blood cortisol levels are highly correlated. Cortisol level has been frequently used as a stress marker in various studies. Blood cortisol level is known to reach its peak at 20 - 30 min after stress induction [17] [18].

Meanwhile, $\alpha$-amylase is used as a marker of the SAM system. It is one of the digestive enzymes in the saliva and pancreatic fluid, and the salivary glands are known to secrete more $\alpha$-amylase in response to heightened sympathetic activity. In addition, salivary $\alpha$-amylase secretion is augmented via direct nerve stimulation. Therefore, changes in its activity can be determined very early (within a few minutes) as opposed to the changes in hormone levels which take much longer to respond.

Our findings show that the cortisol levels, which take longer to show changes, decreased initially after the exercise while quick-responding $\alpha$-amylase activity increased acutely after training on the Power Plate ${ }^{\circledR}$. These results suggest that sympathetic activity may be suppressed during the early stages of training on the Power Plate ${ }^{\circledR}$, 
but is subsequently activated after approximately $10.5 \mathrm{~min}$. Taken together, while WBV training may promote relaxation initially, it may subsequently induce stress if performed for longer than $10.5 \mathrm{~min}$. In light of these findings, a shorter exercise regimen appears more useful for inducing a relaxation response, but further studies will need to investigate the effect of exercise duration on autonomic functions over time via heart rate variability to determine the autonomic correlates of WBV training. Alternatively, stretching exercises that are more relaxation oriented and their optimal time should be explored as well. Finally, more studies are needed to determine the effect of WBV in the elderly because our participants were all young and in their thirties.

\section{Conclusion}

In our study, we evaluated the biological response to WBV training by measuring cutaneous blood flow and salivary components. We found that WBV appears to regulate autonomic activity, in particular, suppress sympathetic activity and improve bodily functions. Thus WBV exercise may be conducive for stress management, but further investigation is warranted to determine the optimal duration of WBV training for the purpose of alleviating stress.

\section{Acknowledgements}

The authors thank Ms. Tomoko Mizuta at PROTEA Japan Co., LTD for preparing the exercise room and providing instructions on the stretching exercises. We also thank the study participants, investigators, and research support staff at the Department of Hospital Administration and the Center for Advanced Kampo Medicine and Clinical Research at Juntendo University Graduate School of Medicine.

\section{Conflict of Interest}

The authors declare no conflicts of interest.

\section{References}

[1] Perchthaler, D., Horstmann, T. and Grau, S. (2013) Variations in Neuromuscular Activity of Thigh Muscles during Whole-Body Vibration in Consideration of Different Biomechanical Variables. Journal of Sports Science and Medicine, 12, 439-446.

[2] Verschueren, S.M., Roelants, M., Delecluse, C., Swinnen, S., Vanderschueren, D. and Boonens, S. (2004) Effect of 6-Month Whole Body Vibration Training on Hip Density, Muscle Strength, and Postural Control in Post-Menopausal Women: A Randomized Controlled Pilot Study. Journal of Bone and Mineral Research, 19, 352-359. http://dx.doi.org/10.1359/JBMR.0301245

[3] Gusi, N., Raimundo, A. and Leal, A. (2006) Low-Frequency Vibratory Exercise Reduces the Risk of Bone Fracture More than Walking: A Randomized Controlled Trial. BMC Muscoloskeletal Disorders, 7, 92-100. http://dx.doi.org/10.1186/1471-2474-7-92

[4] Slatkovska, L., Alibhai, S.M., Beyene, J. and Cheung, A.M. (2010) Effect of Whole-Body Vibration on BWD: A Systematic Review and Meta-Analysis. Osteoporosis International, 21, 1969-1980. http://dx.doi.org/10.1007/s00198-010-1228-z

[5] Merriman, H. and Jackson, K. (2009) The Effects of Whole-Body Vibration Training in Aging Adults: A Systematic Review. Journal of Geriatric Physical Therapy, 32, 134-145. http://dx.doi.org/10.1519/00139143-200932030-00009

[6] Vanes, I.J., Geurts, A.C., Hendricks, H.T. and Duysens, J. (2004) Short-Term Effects of Whole-Body Vibration on Postural Control in Unilateral Chronic Stroke Patients: Preliminary Evidence. American Journal of Physical Medicine \& Rehabilitation, 83, 867-873. http://dx.doi.org/10.1097/01.PHM.0000140801.23135.09

[7] Chan, K.S., Liu, C.W., Chen, T.W., Weng, M.C., Huang, M.H. and Chen, C.H. (2012) Effects of a Single Session of Whole Body Vibration on Ankle Plantarflexion Spasticity and Gait Performance in Patients with Chronic Stroke: A Randomized Controlled Trial. Clinical Rehabilitation, 26, 1087-1095. http://dx.doi.org/10.1177/0269215512446314

[8] Ruck, J., Chabot, G. and Rauch, F. (2010) Vibration Treatment in Cerebral Palsy: A Randomized Controlled Pilot Study. Journal of Musculoskeletal and Neuronal Interactions, 10, 77-83.

[9] Wunderer, K., Schabrun, S.M. and Chipchase, L.S. (2010) Effects of Whole Body Vibration on Strength and Functional Mobility in Multiple Sclerosis. Physiotherapy Theory and Practice, 26, 374-384.

http://dx.doi.org/10.3109/09593980903147384

[10] Tubanski, S., Haas, C.T., Schmidtbleicher, D., et al. (2005) Effects of Random Whole-Body Vibration on Postural 
Control in Parkinson's Disease. Research in Sports Medicine, 13, 243-256. http://dx.doi.org/10.1080/15438620500222588

[11] Haas, C.T., Turbanski, S., Kessler, K. and Schmidtbleicher, D. (2006) The Effect of Random Whole-Body Vibration on Motor Symptoms in Parkinson's Disease. NeuroRehabilitation, 21, 29-36.

[12] Ebersbach, G., Edler, D., Kaufhold, O. and Wissel, J. (2008) Whole Body Vibration versus Conventional Physiotherapy to Improve Balance and Gait in Parkinson's Disease. Archives of Physical Medicine and Rehabilitation, 89, 399403. http://dx.doi.org/10.1016/j.apmr.2007.09.031

[13] Izawa, S., Shirotsuki, K., Sugaya, N., Ogawa, N., Suzuki, K. and Nomura, S. (2007) The Application of Saliva to an Assesment of Stress: Procedures for Collecting and Analyzing Salivary Substances. Japanese Journal of Complementary and Alternative Medicine, 4, 91-101. http://dx.doi.org/10.1625/jcam.4.91

[14] Bosco, C., Iacovelli, M., Tsarpela, O., Cardinale, M., Bonifazi, M., Tihanyi, J., Viru, M., De Lorenzo, A. and Viru, A. (2000) Hormonal Responses to Whole-Body Vibration in Men. European Journal of Applied Physiology, 81, 449-454. http://dx.doi.org/10.1007/s004210050067

[15] Selye, H. (1936) A Syndrome Produced by Diverse Noxious Agents. Nature, 138, 32-32. http://dx.doi.org/10.1038/138032a0

[16] Robert, J.G., Andrew, B. and David, S.K. (1989) An Introduction to Health Psychology. McGraw-Hill, New York.

[17] Kirschbaum, C. and Hellhammer, D.H. (1994) Salivary Cortisol in Psychoneuroendocrine Research. Psychoneuroendocrinology, 19, 313-333. http://dx.doi.org/10.1016/0306-4530(94)90013-2

[18] Kirschbaum, C. and Hellhammer, D.H. (1989) Salivary Cortisol in Psychobiological Research: An Overview. Neuropsychobiology, 22, 150-169. http://dx.doi.org/10.1159/000118611 